\title{
Hirschsprung's disease, hypoplastic nails, and minor dysmorphic features: a distinct autosomal recessive syndrome?
}

\author{
L I AL-GAZALI*, D DONNAI†, AND R F MUELLER* \\ From ${ }^{*}$ the Department of Genetic Counselling, The Clarendon Wing, The General Infirmary at Leeds, Leeds \\ LS2 9NS; and the Department of Medical Genetics, St Mary's Hospital, Manchester M13 0JH.
}

SUMmARY Three children are reported, a male and female sib pair of Sikh origin and a male child who was the offspring of first cousin Pakistani Muslims, with Hirschsprung's disease, hypoplastic nails, and similar minor dysmorphic features. We consider that they represent a distinct autosomal recessive syndrome.

We report three children with Hirschsprung's disease, hypoplastic nails, and minor dysmorphic features. The features resemble those seen in the children described by Rudiger $e a^{1}$ and the children who have the autosomal recessive syndrome described by Fryns et al. ${ }^{2-7}$ We believe, however, that our cases have a distinct autosomal recessive syndrome.

\section{Case reports}

\section{CASE 1}

A male infant was the third child born to first cousin Pakistani Muslim parents. Bilateral hydronephrosis was noted on routine ultrasound examination in mid-trimester. Delivery at 38 weeks was normal, birth weight $3050 \mathrm{~g}$, head circumference $34 \mathrm{~cm}$. An imperforate anus was noted for which he received an anal cutback. A vesicostomy was performed for an obstructed bladder thought to be the result of posterior urethral valves. Craniofacial dysmorphic features noted were loose skin, a narrow bitemporal region, upward slanting palpebral fissures, a fleshy groove under the eyes, a narrow philtral groove, a V shaped indentation in both vermilion borders, a narrow, high arched palate, micrognathia, and prominent ear lobes with abnormal helices (fig 1). The fingers of both hands had small terminal phalanges with absent nails on the fifth fingers and hypoplastic nails on the other fingers and all the toes (fig 2).

He subsequently developed abdominal distension. A barium enema and rectal biopsy showed

Received for publication 20 October 1987.

Revised version accepted for publication 2 December 1987 short segment Hirschsprung's disease and a right transverse colostomy was performed. A left inguinal hernia was repaired. An IVP showed right hydronephrosis with delay at the pelviureteric junction and an irregular calyceal pattern of the left kidnes

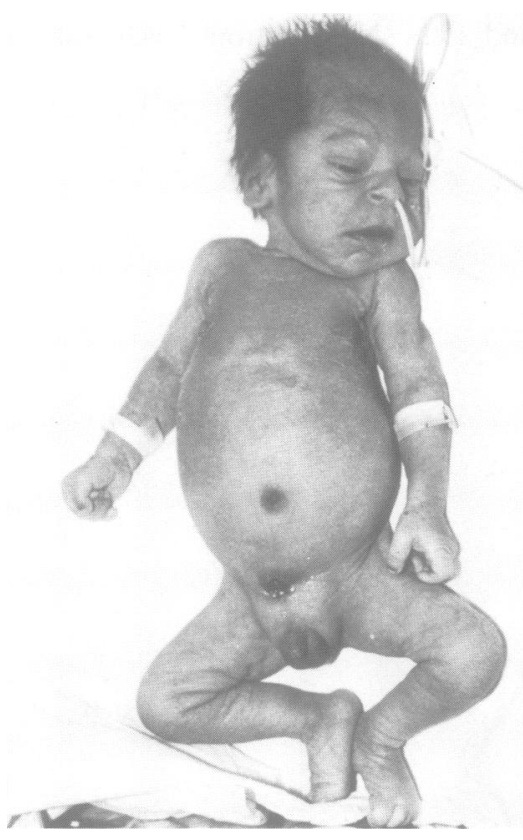

FIG 1 Case 1 showing micrognathia, low set ears with abnormal helices, and grooves under eyes. Also note distended abdomen, vesicostomy, and left inguinal hernia. 


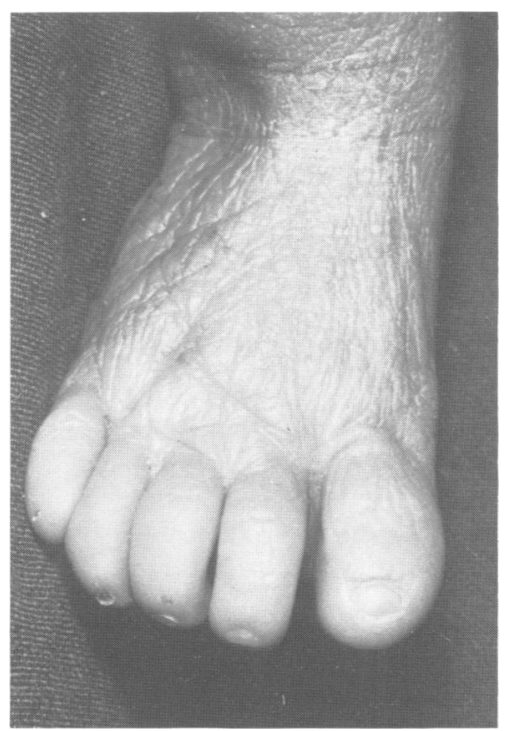

FIG 2 Right foot of case 1 showing hypoplastic nails.

He showed delayed development and abnormal neurological findings and died at home suddenly at the age of two months. Blood lymphocyte chromosomes were normal male, $46, X Y$. No necropsy was performed.

\section{CASE 2}

A male infant was the second child born by normal delivery to unrelated parents of Sikh extraction after a normal pregnancy, birth weight 2700 g. Dysmorphic features noted in the newborn period included low set ears, large anterior fontanelle, a flat facies, high arched palate, short neck, clawed hands with overlapping fingers, rocker bottom feet, an undescended left testis, and hyperconvex toe nails. He developed abdominal distension with bile stained vomiting and did not pass meconium. A presumed diagnosis of trisomy 18 was made and the child died aged six days. The lymphocyte chromosomes were normal male, $46, X Y$. No necropsy was performed.

\section{CASE 3}

In the next pregnancy of the same couple, a female child was born by normal delivery at term. Birth weight and head circumference were on the 3rd centile. Dysmorphic facial features noted in the newborn period included low set, cup shaped ears with a thin outer helix, micrognathia, and a deep transverse groove on the chin (fig 3). Other dysmorphic features included overlapping fingers which were hypoplastic distally, hypoplastic finger and toe nails with an absent fourth left finger nail (fig 4), and rocker bottom feet with an 'appended' fifth toe bilaterally. She developed bile stained vomiting on the second day and did not pass meconium. A laparotomy showed tenacious meconium in the

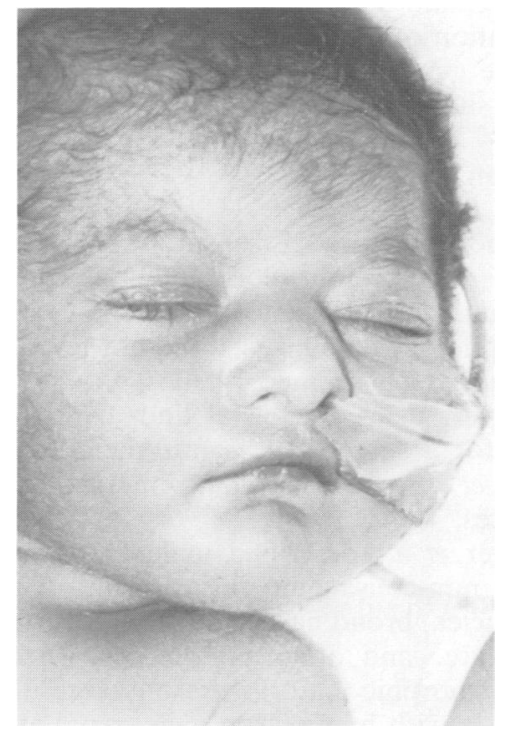

FIG 3 Facies of case 3 showing similar features to case 1 with grooves under the eyes and deep transverse crease on the chin.

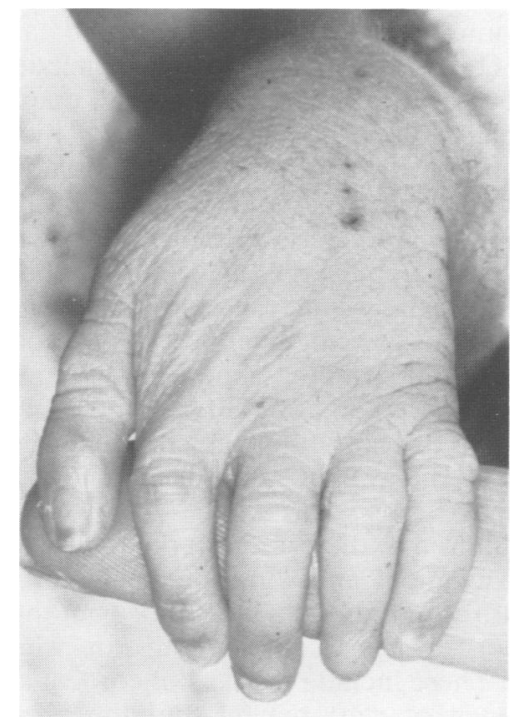

FIG 4 Left hand of case 3 showing hypoplasia of distal phalanges and nails. 
terminal ileum and a biopsy revealed Hirschsprung's disease. Postoperatively a heart murmur was noted. She developed jaundice, fits, and signs of septicaemia and died aged eight days. Necropsy showed long segment Hirschsprung's disease, extending into the ileum, absence of lung lobulation, a small VSD and PDA, and minor renal pelvicalyceal dilatation. Examination of the brain showed a mild abnormality of the gyral pattern. Lymphocyte chromosomal analysis showed a normal female pattern, 46,XX.

\section{Discussion}

While the finding of Hirschsprung's disease in the children in the present report might be considered a good 'handle' for a diagnostic search of the London Dysmorphology database, ${ }^{8}$ it did not reveal a single syndrome which seemed likely.

However, using the distal limb hypoplasia as a feature for a search, the syndromes described by Rudiger et $a l^{1}$ and Fryns et al ${ }^{2}$ emerged as possible candidates.

Rudiger et al $^{1}$ described male and female sibs, born to non-consanguineous parents, who had a coarse facies, broad nasal bridge, anteverted nares, cleft palate, and abnormal helices. In addition, other dysmorphic features present were distal limb hypoplasia with hypoplastic nails, renal abnormalities (hydronephrosis), and genital abnormalities (bicornuate uterus in the female).

Fryns et $a l^{2}$ described two female sibs with a history in pregnancy of polyhydramnios. They had a coarse facies, corneal clouding, broad nasal bridge, macrostomia with a small jaw, and abnormal helices. Other dysmorphic features included distal limb hypoplasia with hypoplastic nasil, diaphragmatic hernia with probable secondary hypoplastic lungs, absent or abnormal lung lobulation, and genital and central nervous system abnormalities. Subsequently Goddeeris $e t a l^{3}$ reported a further female child, Lubinsky et $a l^{4}$ reported a male and female sib pair, Meinecke and Fryns ${ }^{5}$ a further female child of consanguineous parents, and Young et a ${ }^{6}$ a male child with the same features, constituting what is now accepted as the Fryns syndrome. ${ }^{7}$ There is discussion as to whether the children reported by Rudiger et $a^{1}$ might also have the syndrome reported by Fryns et al. ${ }^{2}$

The children in the present report share the distal limb involvement and some of the minor dysmorphic features in these two syndromes but there were a number of significant findings in which they differed. These include the lack of corneal clouding, the absence of cleft palate, and the presence of Hirschsprung's disease (table).

Curry and $\mathrm{Hall}^{9}$ also observed a male and female
TABLE Clinical features.

\begin{tabular}{|c|c|c|c|c|c|c|c|}
\hline & \multirow{2}{*}{$\begin{array}{l}\text { Fryns } \\
\text { et } a t^{2}\end{array}$} & \multicolumn{3}{|c|}{ Present report } & \multirow{2}{*}{$\begin{array}{l}\text { Rudiger } \\
\text { et al }\end{array}$} & \multirow{2}{*}{$\begin{array}{l}\text { Curry } \\
\text { and } \\
\text { Hall }^{9}\end{array}$} & OS \\
\hline & & 1 & 2 & 3 & & & \\
\hline \multicolumn{7}{|l|}{ General } & \\
\hline Sex & $2 \mathrm{M}: 5 \mathrm{~F}$ & $\mathbf{M}$ & $\mathbf{M}$ & $\mathbf{F}$ & 1M:1F & 1M:1F & $\frac{\overrightarrow{7}}{9}$ \\
\hline Polyhydramnios & $4 / 7$ & - & - & - & $0 / 2$ & $? ?$ & อ \\
\hline \multicolumn{7}{|l|}{ Craniofacial } & \\
\hline Coarse facies & $3 / 4$ & - & - & - & $2 / 2$ & $0 / 2$ & $\bar{\omega}$ \\
\hline Corneal clouding & $3 / 6$ & - & - & - & $0 / 2$ & $0 / 2$ & $\vec{r}$ \\
\hline Broad nasal bridge & $4 / 5$ & - & $?$ & - & $2 / 2$ & $0 / 2$ & \\
\hline Large, anteverted nares & $3 / 6$ & + & $?$ & - & $2 / 2$ & $2 / 2$ & \\
\hline Macrostomia & $3 / 5$ & + & $?$ & - & $0 / 2$ & $0 / 2$ & \\
\hline Micrognathia & $6 / 7$ & + & $?$ & - & $0 / 2$ & $0 / 2$ & \\
\hline Cleft palate & $6 / 7$ & - & $?$ & - & $2 / 2$ & $2 / 2$ & $\overline{3}$ \\
\hline Abnormal helices & $5 / 6$ & + & $?$ & + & $2 / 2$ & $0 / 2$ & \\
\hline Short neck & $5 / 7$ & + & $?$ & - & $2 / 2$ & $0 / 2$ & \\
\hline \multicolumn{7}{|l|}{ Limb } & \\
\hline \multicolumn{7}{|l|}{ Distal limb } & \\
\hline hypoplasia & $5 / 5$ & + & + & + & $2 / 2$ & $2 / 2$ & \\
\hline Hypoplastic nails & $6 / 7$ & + & + & + & $2 / 2$ & $2 / 2$ & \\
\hline Camptodactyly & $2 / 7$ & - & $?$ & - & $0 / 2$ & $0 / 2$ & \\
\hline \multicolumn{7}{|l|}{ Visceral } & \\
\hline Diaphragmatic defect & $6 / 7$ & - & - & - & $0 / 2$ & $0 / 2$ & \\
\hline Lung hypoplasia & $7 / 7$ & - & - & - & $0 / 2$ & $0 / 2$ & \\
\hline Absent/abnormal & & & & & & & \\
\hline lung lobulation & $4 / 6$ & - & $?$ & + & $1 / 2$ & $?$ & \\
\hline Hirschsprung's disease & $0 / 7$ & + & $(+)$ & + & $0 / 2$ & $2 / 2$ & \\
\hline Renal abnormalities & $2 / 4$ & + & $?$ & + & $2 / 2$ & $1 / 2$ & (D) \\
\hline Genital abnormalities & $4 / 5$ & - & $?$ & - & $1 / 2$ & $0 / 2$ & \\
\hline CNS abnormalities & $5 / 6$ & $?$ & $?$ & $(+)$ & $0 / 2$ & $? / 2$ & \\
\hline
\end{tabular}

sib pair born to non-consanguineous parents wit Hirschsprung's disease, cleft palate, digital abno malities, mental retardation, and growth deficiency. They differed from the present cases in having cleft palate and having survived the neonatal/infant period.

Although the distal limb involvement in these children could be consistent with the teratogenic effects of phenytoin, neither mother took any medication during pregnancy.

While it could be postulated that the children reported in all three candidate syndromes were, in fact, phenotypic variants of the same autosomal recessive syndrome, this seems unlikely in view of previous experience with other dysmorphic syndromes. We feel that the unique features seen in the three children in the present report, namely Hirschsprung's disease and distal limb hypoplasia, represent a separate, probably autosomal recessive, syndrome.

\section{References}

${ }^{1}$ Rudiger RA, Schmidt W, Loose DA, Passarge E. Severe developmental failure with coarse facial features, distal limb hypoplasia, thickened palmar creases, bifid uvula, and ureteral stenosis: a previously unidentified familial disorder with lethal outcome. J Pediatr 1971;79:977-81.

2 Fryns JP, Moerman F, Goddeeris P, Bossuyt C, Van den Berghe H. A new lethal syndrome with cloudy corneae, diaphragmatic defects and distal limb deformities. Hum Genet 1979;50:65-70.

\section{.}


${ }^{3}$ Goddeeris P, Fryns JP, Van den Berghe H. Diaphragmatic defects, craniofacial dysmorphism, cleft palate and distal limb deformities-a new lethal syndrome. J Genet Hum 1980;28: $57-60$.

${ }^{4}$ Lubinsky M, Severn C, Rapoport JM. Fryns syndrome: a new variable multiple congenital anomaly (MCA) syndrome. Am J Med Genet 1983;14:461-6.

${ }^{5}$ Meinecke P, Fryns JP. The Fryns syndrome: diaphragmatic defects, craniofacial dysmorphism, and distal digital hypoplasia. Clin Genet 1985;28:516-20.

6 Young ID, Simpson K, Winter RM. A case of Fryns syndrome. J Med Genet 1986;23:82-4.

${ }^{7}$ Fryns JP. Fryns syndrome: a variable MCA syndrome with diaphragmatic defects, coarse face, and distal limb hypoplasia $J$ Med Genet 1987;24:271-4.

8 Winter RM, Baraitser M. The London Dysmorphology Database. J Med Genet 1987;24:509-10.

${ }^{9}$ Curry CJ, Hall BD. A new familial syndrome with Hirschsprung's disease, cleft palate, digital abnormalities, mental retardation and growth deficiency. Clin Res 1979;27:118.

Correspondence and requests for reprints to Dr L I Al-Gazali, Department of Genetic Counselling, Clarendon Wing, The General Infirmary, Belmont Grove, Leeds LS2 9NS. 